\title{
La construcción de la educación laica en Argentina, Brasil y México $(1824-1879)^{1}$
}

\section{The construction of lay education in Argentina, Brazil, and Mexico (1824-1879)}

Alejandro Ortiz Cirilo

Resumen

En el presente trabajo son reconstruidos los procesos de conformación de los regímenes de laicidad educativa de Argentina, Brasil y México, poniendo énfasis en las distintas etapas presentes en la construcción de sus sistemas educativos. Son retomados las especificidades de las escuelas públicas y las privadas, en los diferentes niveles educativos y en diversos contextos históricos, focalizando en los aspectos jurídicos que sirvieron de normativa.

Palabras clave: Educación laica; educación religiosa; relaciones Estado-Iglesia; secularización.

\begin{abstract}
This paper reconstructs the processes of conformation of the educational laicity regimes of Argentina, Brazil and Mexico, emphasizing the different stages present in the construction of their educational systems. The specificities of public and private schools, at different educational levels and in different historical contexts, are taken up again, focusing on the legal aspects that served as norms.
\end{abstract}

Keywords: lay education; religious education; church and state; secularization

\section{Introducción}

El objetivo de este artículo es reconstruir los procesos que siguieron los diferentes regímenes de laicidad educativa en Argentina, Brasil y

1 Trabajo recibido el 22/04/2019. Aceptado el 28/06/2019.

${ }^{2}$ Universidad Autónoma de la Ciudad de México. Contacto: aortizc1985@gmail.com 
México. Se pone énfasis en las diversas etapas que se suscitaron como parte del proceso de construcción de sus sistemas educativos. La primera de ellas se ubica con la promulgación de las primeras constituciones políticas latinoamericanas en las que se protegió al catolicismo como religión oficial y se estableció de manera formal la intolerancia religiosa. Una segunda etapa se inicia con la progresiva secularización de mediados del siglo XIX cuando los Estados latinoamericanos comenzaron a tomar el control sobre las actividades educativas y la enseñanza religiosa empezó a ser relegada de los planes y programas oficiales. La tercera etapa contempla los intentos de los gobiernos liberales que integraron nuevas leyes generales que trascendieron al ámbito escolar.

A lo largo del trabajo se retoman los elementos que incluyeron específicamente a las escuelas públicas y las privadas, en sus diferentes niveles de estudios. Se exponen las razones en sus distintos contextos históricos y se enfoca en los aspectos jurídicos como marco para orientar y normar las relaciones sociales. Por último, se contrastan los espacios, público/privado, religioso/secular/laico. El trabajo se basa en fuentes de información primaria como leyes, reglamentos, decretos y acuerdos. Se utilizaron, asimismo, fuentes secundarias para explicar brevemente el contexto histórico en cada caso.

\section{De la intolerancia religiosa en los albores de los Estados latinoameri- canos}

Con la emancipación de los nuevos Estados latinoamericanos se buscó el reconocimiento, legitimidad y construcción de una identidad propia que se afirmara en relación con otros Estados soberanos. En todo este proceso ¿Qué papel se le asignó a la religión católica en las primeras constituciones políticas de Argentina, Brasil y México?¿Cómo influyó el establecimiento de una religión oficial en la instrucción pública de estos países? En las primeras Constituciones políticas latinoamericanas se plantearon cuestiones fundamentales con relación al tipo de régimen que se debía establecer, sobre elementos vinculados con la libertad e independencia política del dominio extranjero, pero también con relación al establecimiento del catolicismo como religión oficial. Este fue uno de los elementos que conservaron prácticamente todos los recién formados Estados latinoamericanos. Adicionalmente, se negó el derecho a profesar y ejercer públicamente cualquier otro culto. En 1824 Brasil promul- 
gó su primera Carta Magna y decretó que el catolicismo era la religión oficial del imperio. Se permitió la práctica de otros cultos, pero con la prescripción de que solo podría hacerse de forma privada, al interior de las casas o dentro de los templos. Sobre este aspecto México fue mucho más drástico, pues su primera Constitución, también promulgada en 1824, instituyó de manera formal la intolerancia religiosa y prohibió de forma expresa el ejercicio de cualquier otra religión. Por su parte, la Constitución argentina de 1826 exigió que los habitantes respetaran a la religión católica sin importar sus opiniones religiosas.

Ahora bien, a pesar de que en las primeras Constituciones políticas de Argentina, Brasil y México se estableció un trato preferencial para la Iglesia católica, esto no se tradujo en una sumisión total del Estado a la autoridad eclesiástica, ni se mantuvo un paralelismo entre los poderes estatales y religiosos. De hecho, en estos países se establecieron ciertos controles sobre las actividades eclesiásticas. El artículo 95 de la Constitución argentina establecía que era facultad del ejecutivo ejercer el patronato general respecto a las iglesias, beneficios y personas eclesiásticas, con arreglo a las leyes, así como nombrar a los arzobispos y obispos a propuesta en terna del Senado. En el caso de Brasil, el artículo 102 (II) de su Constitución le concedió al Poder Ejecutivo amplias facultades para nombrar obispos y promover beneficios eclesiásticos. En México la Constitución no le otorgó al presidente facultades sobre los ministros de culto. No obstante, el artículo 110 dispuso que, en conjunto con el Congreso General, podía conceder el pase o retener decretos conciliares, bulas pontificias, breves y rescriptos. Así es posible observar procesos de cambio que aparentemente eran contradictorios, ya que por un lado se protegió al catolicismo como religión oficial y se estableció la intolerancia religiosa, pero al mismo tiempo se fueron estableciendo ciertos controles sobre las actividades de la Iglesia católica, lo que en cierta medida fue un factor importante para los procesos de secularización que se fueron suscitando en décadas posteriores.

\section{La intervención estatal en la instrucción pública}

En las primeras Constituciones de Argentina, Brasil y México el tema de la instrucción pública fue fundamental. Las élites locales buscaron crear instrumentos para la formación de ciudadanos bajo el control del Estado. Para cumplir este ropósito, se buscó crear una nueva forma 
de organización política que incluyera a las masas populares y dejara la instrucción primaria bajo control del Estado y, ya no, de la Iglesia católica.

De acuerdo con Anne Staples (1988), la construcción de los nuevos Estados nacionales también se articuló con la formación de ciudadanos a través de la lectura. No obstante, es necesario señalar que en sus inicios la formación de la ciudadanía se vinculó estrechamente con la dimensión religiosa impulsada por el catolicismo. Esto fue cambiando cuando se comenzaron a integrar visiones liberales y con la progresiva secularización de las sociedades latinoamericanas de mediados del siglo XIX (Sábato, 1999). Cuando se entró en una nueva etapa en la que las autoridades estatales comenzaron a tomar el control de la educación, se inició una discusión en torno al papel de la escuela como un elemento central de cambio en la sociedad. Las disposiciones establecidas en la legislación de cada país posibilitaron que los Estados nacionales comenzaran a ganar atribuciones por encima de las instituciones religiosas.

La Constitución del imperio de Brasil de 1824 en su artículo 179 inciso 32 estableció que la instrucción primaria sería gratuita para todos los ciudadanos. No obstante, durante esa época el panorama educativo no era alentador, la mayor parte de la población era analfabeta y la sociedad seguía siendo esclavista. Este es un punto importante, ya que hasta 1840 -cuando Pedro II fue proclamado emperador a causa de la inestabilidad política que predominaba entre distintas facciones de liberales y conservadores ${ }^{3}$ los esclavos no eran reconocidos como ciudadanos y, por lo tanto, tampoco eran sujetos de derechos civiles y políticos (Carvalho, 1995). Al iniciar su mandato, el emperador siguió enfrentando diversos problemas relacionados con la pretendida regulación del Estado sobre todas las actividades religiosas. Este derecho se había extendido a cada una de las provincias mediante una enmienda constitucional conocida como Ato Adicional (1834) y además se publicaron leyes, decretos, reglamentos y regulaciones adoptadas por el gobierno imperial, lo que provocó diferencias con la Iglesia católica (Thornton, 1948).

En tanto, en México la primera Constitución Federal de 1824 hay una única referencia relativa a la educación en el artículo 50, en el que se le otorgó al Congreso General la facultad exclusiva de promover la ilus-

\footnotetext{
${ }^{3}$ Pedro I abdicó en 1831 dejando la corona a cargo de su hijo de seis años, como no tenía edad suficiente para suceder en el trono, un comité temporal funcionó como regencia hasta 1840 .
} 
tración. En virtud de su carácter federal, cada provincia tenía la facultad de normar lo relativo a la instrucción pública en sus territorios. A partir de este momento y hasta 1847 fueron generados cambios en la legislación nacional que se caracterizaron por una completa desarticulación entre el gobierno nacional y los estatales (Vázquez, 1993).

Después de la Revolución de 1810 en Argentina se suscitaron diversos cambios en la política y la enseñanza. La orientación educativa osciló entre el liberalismo y el mantenimiento de la enseñanza religiosa. Durante el prolongado periodo en el que Juan Manuel de Rosas estuvo frente de la Confederación (1830-1852) se estableció un gobierno caudillista que reimplantó la educación religiosa obligatoria y exigió, por parte de la población general y de los docentes en particular, una firme devoción a su causa y a su persona (Delgado Criado, 1992).

Dentro de todo este conjunto de normas se puede apreciar un gradual debilitamiento de la Iglesia católica y un fortalecimiento del Estado en materia educativa. Se expandieron sus atribuciones y esto incluyó de manera importante su predominio sobre las instituciones educativas de carácter confesional. Hasta este momento no había una separación formal entre el Estado y la Iglesia sino un intento del Estado por incidir en la educación.

\section{El dilema entre la libertad religiosa o la continuidad del orden cató- lico}

Atendiendo al problema de la enseñanza en Brasil, en 1851 el emperador Pedro II autorizó una reforma en el municipio de Corte, que fue reglamentada por el ministro Luiz Pedreira do Couto Ferraz. El Decreto de Ley 1.331 (1854) introdujo cambios en la enseñanza primaria y secundaria con relación a las facultades del Inspector General de Educación en esta región. Un aspecto importante del contenido de esta reforma fue el relativo a la libertad de enseñanza. El gobierno se adjudicó la facultad para permitir que la iniciativa privada, compuesta por una amplia mayoría de congregaciones religiosas pudiera gozar de la libertad para abrir escuelas y ofrecer enseñanza primaria y secundaria. Con la reforma nadie podía abrir una institución escolar sin previa autorización del Inspector General. A pesar de esto, mantuvo sin cambios los contenidos religiosos en la escuela. La novedad de este decreto fue que obligó a los directores de las escuelas privadas a contar con un sacerdote 
para los alumnos que no fuesen católicos. Esta fue la primera ocasión que la legislación educativa en Brasil reconoció la existencia de estudiantes ajenos a la comunión católica. Un año más tarde, en el municipio de Corte, se reforzó esta medida reconociendo el derecho de los alumnos a no asistir a este tipo de clases. Además, se dispuso que, en todo caso, tendrían que impartirse en determinados días de la semana y siempre antes o después de las horas destinadas a otras disciplinas. Esto marcó un cambio de las relaciones entre el Estado y la Iglesia y fue un punto importante que fortaleció las competencias del Estado brasileño en materia educativa.

Siguiendo con los procesos de cambio, a mediados del siglo XIX en Argentina y México se promulgaron nuevas Constituciones y, a pesar de que se conservaron ciertos privilegios para la Iglesia católica, en cierta medida, recibieron el impulso de las ideas liberales de la época. Se integraron nuevos elementos tendientes a garantizar el ejercicio de las libertades y se comenzó a dejar de lado la pretendida idea de una uniformidad católica como eje de la unidad y la identidad nacional.

Después del derrocamiento de Juan Manuel de Rosas, en Argentina se comenzó la reconstrucción y reorganización de las instituciones políticas. Un primer paso fue la celebración del Congreso Constituyente que se reunió en la Ciudad de Santa Fe en 1853. De acuerdo con Campobassi (1961) esa asamblea estuvo influenciada, en materia religiosa, por diversas corrientes ideológicas y al final, predominó una corriente liberal progresista o innovadora, encarnada en los pensamientos, escritos y proyectos de Echeverría, Sarmiento, Mitre, García Gutiérrez y otros, que defendieron la implantación de la libertad de cultos y la eliminación de toda religión de Estado. En esta nueva Constitución se mantuvo el sostenimiento del culto católico en el artículo $2^{\circ}$, pese a lo cual, y de acuerdo con los legisladores de la época, el sostenimiento del culto católico no implicaba la adopción de una religión de Estado sino solo una referencia a la tradición católica de la sociedad argentina. Asimismo, se proclamó la libertad de cultos, lo que representó uno de los cambios más importantes. No obstante, entre las funciones del Congreso nacional, se dispuso que debería promover la conversión de los indios al catolicismo.

En materia educativa la Constitución argentina de 1853 obligó a las provincias a implantar un sistema de enseñanza primaria gratuita. Sin embargo, este precepto se suprimió con la reforma de 1860. En consecuencia, a las provincias les resultó costoso el mantenimiento de la enseñanza primaria. De modo que se generó una política de subvencio- 
nes que trató de frenar su impotencia financiera (Miranda, 1981). El artículo $5^{\circ}$ de la Constitución le concedió a cada una de las provincias la posibilidad de crear su propia carta magna y, al mismo tiempo, les otorgó cierta autonomía para legislar en materia educativa, pero dado que estaban subordinadas a la Constitución Nacional, debían permitir el libre ejercicio del culto y garantizar la libertad de enseñanza. Las provincias pusieron la enseñanza bajo la dirección de las municipalidades, pero unificada la nación argentina se consideró que la enseñanza primaria era tarea común de la nación y las provincias. Para suplir los escasos recursos provinciales, el Congreso otorgó subsidios a través de la Ley del 24 de septiembre de 1857 de la Confederación, y sucesivos decretos a partir de 1864; ley 463 de 1871 (Zorraquín, 1996). Una lectura de lo importante que fue este artículo, no solo en términos económicos, nos remite a considerar que toda la legislación secundaria, leyes generales, y reglamentos, debían alinearse a este precepto, lo que también incluyó la instrucción pública.

En el caso de México, después de un prolongado proceso de discusiones legislativas, el 5 de febrero de 1857 se promulgó una nueva Constitución política. Los cambios que se integraron atendieron diversos aspectos. En primer lugar, se omitió el preámbulo que hacía referencia Dios, lo que no solo fue una cuestión de forma, pues esta breve sección adquiere una connotación simbólica. Un segundo aspecto importante fue que ya no se hizo mención a tratamientos privilegiados a la Iglesia católica. Asimismo, se abrogó la intolerancia hacía otras religiones. En tercer lugar, se contempló la autoridad del pueblo, lo que significó un cambio sustancial porque, de cierta forma, se reconoció que la soberanía no provenía de una fuente de carácter sagrado. El artículo 40 la Constitución mexicana de 1857 estableció que era voluntad del pueblo mexicano constituirse en una república representativa, democrática, federal, compuesta de estados libres y soberanos en todo lo concerniente a su régimen interior, pero unidos en una federación establecida según los principios de esta ley fundamental.

En materia educativa el artículo $3^{\circ}$ dispuso que la enseñanza era libre. La ley determinaría qué profesiones necesitaban título para su ejercicio y con qué requisitos se debían expedir. Ahora, es importante considerar las diferencias conceptuales entre la educación libre y la educación laica, puesto que ambas se han asimilado de forma indistinta para referir a un tipo de educación ajena a la influencia del clero e independiente de ideologías dogmáticas. Por un lado, la enseñanza libre no implicó la de- 
rogación de contenidos religiosos, incluso, este tipo de materias quedaron contempladas dentro de los programas oficiales en los tres países. Por otro lado, la educación laica implicó, en un primer momento, la omisión y posteriormente, la prohibición de la religión como centro de toda la enseñanza en las escuelas oficiales y dentro de los horarios de clase. El problema de fondo fue el control que el Estado asumió para regular todas las actividades educativas tanto públicas como privadas.

La declaratoria de educación libre fue una apertura hacia la participación de la sociedad civil, los particulares laicos a ofrecer servicios educativos, sumándose con ello y combatiendo la oferta tradicional de las corporaciones de maestros, las congregaciones religiosas y seglares católicos sólo que con la condición de colocarse bajo la égida del poder estatal (Estrada, 1984).

El Papa Pío IX estimó que la relación de la Iglesia católica romana con las sociedades americanas se diluía a causa de la legislación liberal. Por esta razón, en 1864 manifestó su reprobación enérgica mediante la publicación del Syllabus Errorum, un catálogo que comprendía «los principales errores de nuestra época». En la proposición 47 consideró como un error que los establecimientos públicos dedicados a la enseñanza quedaran al margen de la autoridad de la Iglesia y sujetos al pleno arbitrio de la autoridad civil y política según el criterio de los gobernantes y de acuerdo con las ideas comunes de la época. En la proposición 48 señaló como error que los católicos pudieran aprobar un sistema educativo de la juventud que no tuviera conexión con la fe católica ni con el poder de la iglesia. La cohesión de los fieles por vía de la censura de los principios liberales no pareció haber tenido gran efecto: en las últimas décadas del siglo XIX, en México, al igual que en el mundo occidental, hubo distintas maneras de ser católico (Cárdenas Ayala, 2015)

\section{Leyes reglamentarias relativas a la enseñanza laica}

Las leyes reglamentarias de educación fueron un antecedente fundamental de la construcción de la laicidad educativa en Argentina, Brasil y México. La enseñanza religiosa había quedado establecida de forma expresa en los planes y programas educativos de estos países. Un punto de inflexión se produjo cuando la religión dejo de ser el centro de toda la enseñanza impartida en las escuelas oficiales $y$, posteriormente cuando se suprimieron los contenidos religiosos del currículum oficial. 
En México, el 15 abril de 1861, el presidente Juárez promulgó la Ley Orgánica de Instrucción Pública que normaba lo referente a la instrucción primaria, secundaria, escuelas especiales, exámenes, catedrático y fondos para la instrucción en el Distrito Federal y territorios. En esta ley ya no se incluyó a la religión en los contenidos de primaria financiadas por los poderes públicos y se creó «un establecimiento modelo» para formar a los profesores de primaria, cuyo programa tampoco incluía contenidos religiosos. En el programa de preparatoria (equivalente a la actual secundaria y preparatoria) ya no se incluyeron las materias «elementos de historia sagrada» ni «religión y filosofía moral» ni ninguna otra asignatura con temas religiosos» (González Villarreal y Arredondo, 2017). Para el 19 de agosto de 1861 se promulgó la Ley de Instrucción Pública de Tamaulipas en la que se declaró la obligación del Estado de sostener y dirigir la enseñanza escolar, de carácter obligatoria, gratuita y laica. Esta fue la primera ley reglamentaria que estableció de manera formal el carácter laico de la educación en dos estados de la república mexicana. A pesar de ser un cambio fundamental, no tuvo carácter federal.

En tanto que en México se libraba una batalla contra la intervención francesa, en Brasil José Liberato Barroso, de orientación liberal, presentó un proyecto de educación para reformular el plan de estudios de la escuela primaria y precisaba que esta debía estar descatolizado pero no descristianizado (Cunha, 2017).Su propuesta defendía la libertad de consciencia, se posicionó contra el sectarismo y defendió que los profesores debían enseñar a los alumnos principios de moral cristiana, pero fuera de los horarios escolares, propuesta que después sería retomada por legisladores argentinos cuando discutieron la Ley 1420 en 1884. Durante la relatoría presentada en la Asamblea General en 1865 se comenzó a discutir y organizar la estructura de su sistema educativo. Entre otros temas, fueron delineadas la instrucción profesional, normal, militar, la educación especial, las sociedades científicas y literarias. A lo largo de las discusiones se argumentó que la Iglesia no tenía derecho de exigir protección del Estado contra alguna libertad de enseñanza; por el contrario, el Estado debía proteger el pleno gozo de la libertad de enseñanza contra las invasiones de la autoridad eclesiástica (Barroso, 1867).

En Argentina también se discutió la importancia de las leyes educativas reglamentarias a nivel provincial. El 20 de julio de 1871, el director de las Escuelas Municipales, Marcos Sastre, presentó el Programa y Reglamento para las Escuelas Municipales. Lo novedoso de esta ley fue 
que omitió la enseñanza de la doctrina católica como parte del programa de estudios vigentes. Incluso, de acuerdo con el artículo $8^{\circ}$, el concepto de moral ya no estaba relacionado con los deberes y virtudes de un creyente, sino con la formación y bienestar del hombre como hijo, como padre, como ciudadano y como trabajador. Estas disposiciones no pasarían desapercibidas por miembros de la Iglesia católica. El 9 de octubre de 1872, el obispo de la Ciudad de Buenos Aires, le escribió al presidente de la municipalidad en reclamo por la decisión de la Dirección de Escuelas de suspender de la enseñanza de la doctrina cristiana en las escuelas municipales. Este reclamo, además de externar el repudio de la Iglesia católica en materia educativa, también era un reflejo de su participación y la defensa de sus intereses por incidir en la formación de las nuevas generaciones.

El 26 de septiembre de 1875 se promulgó la Ley de Instrucción Común de la Provincia de Buenos Aires $N^{\circ}$ 2688. En esta Ley el sistema educativo provincial adquirió un mayor protagonismo. En lo que respecta a la educación religiosa, según el artículo $2^{\circ}$ los padres tutores o personas en cuyo poder se encontraran los niños residentes estaban obligados a darles un minimum de enseñanza religiosa. No obstante, el Consejo General estaba obligado a respetar en la organización de la enseñanza religiosa las creencias de los padres de familia ajenos a la comunión católica. El 30 de septiembre de 1878 se publicó la Ley 934 relativa a la libertad de enseñanza, por la cual se determinó que podrían establecerse escuelas y colegios privados, pero bajo la fiscalización del Estado, medida que muy criticada por la Iglesia católica que pretendía expedir títulos libremente en sus escuelas. En esta ley se asentaron las condiciones para poder presentar un examen general de materias, se delinearon las bases del tribunal especial mixto que sería el encargado de regular toda la actividad de las escuelas particulares y se garantizó el acceso de toda persona a presentar el examen. Por último, se atendió la expedición de certificados. Esta ley fue complementaria de lo dispuesto en la Constitución Nacional de 1853, en la que ya se había establecido la libertad de enseñanza y, pese a las críticas, no se estableció la igualdad de los colegios incorporados a los colegios nacionales oficiales, lo que generó tensiones en grupos conservadores a fines a los proyectos educativos de la Iglesia católica.

El proceso de secularización en México se afirmó a un más, para el 25 de septiembre de 1873 cuando se adicionó al artículo 28 de la Constitución mexicana, según el cual el Estado y la Iglesia eran independien- 
tes entre sí y el Congreso no podía dictar leyes estableciendo o prohibiendo religión alguna (Dublán y Lozano, 1876). Adicionalmente, el artículo $5^{\circ}$ prohibió el establecimiento de órdenes monásticas y el artículo $27^{\circ}$ estableció una prohibición para que las corporaciones religiosas pudieran adquirir o administrar propiedades. Estas reformas fueron trascendentales en materia de libertad de cultos, pero también en lo relativo al papel que jugaba la Iglesia católica en la instrucción pública. Con el establecimiento de nuevas medidas se contemplaron otros elementos entre los que destacó el capítulo $4^{\circ}$ del decreto del Congreso de la Unión en 1874 que estableció:

La instrucción religiosa y las prácticas oficiales de cualquier culto quedan prohibidas en todos los establecimientos de la federación, de los estados y municipios. Se enseñará la moral en los que por la naturaleza de su distinción lo permitan, aunque sin referencia a ningún culto. La infracción de este artículo será castigada con multa y destitución en caso de reincidencia. Las personas que habiten los establecimientos públicos de cualquiera clase pueden, si lo solicitan, concurrir a los templos de su culto y recibir en los mismos establecimientos, en caso de extrema necesidad, los auxilios espirituales de la religión que profesen. En los reglamentos respectivos se fijará la manera de obsequiar esta autorización, sin perjuicio del objeto de los establecimientos y sin contrariar lo dispuesto en el artículo tercero. (Dublán y Lozano, 1876)

En este decreto no solo se omitieron los contenidos religiosos en las escuelas públicas, sino que también se prohibió su ejercicio en su interior y se establecieron sanciones económicas por el incumplimiento de estas disposiciones y destituciones en caso de reincidencia. Este tipo de medidas no se habían implementado anteriormente y fueron resistidos por miembros de la Iglesia católica.

En el contexto en que se produjeron estos cambios no se desconocía la presencia que tenían los colegios particulares. Algunos estaban estrechamente ligados a la Iglesia católica, pero también se contempló que estas reformas tendrían un impacto favorable para la participación de otros agentes educativos con orientaciones distintas al catolicismo. En las disposiciones legales de México, Brasil y Argentina anteriormente expuestas, es posible observar cómo el Estado se fue atribuyendo responsabilidades que no se podían adjudicar y hacer efectivas en los propios establecimientos particulares. En principio, la competencia de estas 
leyes estaba delimitada por la jurisdicción territorial, porque en el caso de Brasil la ley de 1851 solo contemplaba al municipio de Corte, se creó la figura del inspector general y se contempló a los alumnos ajenos a la comunión católica. En tanto que en México la libertad de enseñanza se proclamó por la Constitución federal de 1857 lo que implicó su ejercicio en todos los estados y municipios que conformaban la república mexicana y, en el caso de Argentina, la Ley 934 de 1878 fue complementaria de lo estipulado en su Constitución, por lo que el tema de la enseñanza libre estuvo vinculado con la expedición de títulos por parte de las escuelas privadas.

A finales del siglo XIX en Argentina se fueron sentando las bases para la consolidación de su sistema educativo. Hasta este momento persistían problemas relativos a la falta de maestros y el analfabetismo. Las escuelas se encontraban en una situación precaria e inestable, funcionando en locales inapropiados y sin elementos suficientes (Martínez Paz, 1973). En la 24a sesión ordinaria del 4 de julio de 1883, la Comisión de Culto e Instrucción Pública presentó ante el Congreso Nacional de Argentina el dictamen de la Ley de educación primaria. En este proyecto comenzó a cuestionarse la importancia de la religión en la escuela pública, el artículo $3^{\circ}$ del dictamen presentado señalaba que «Los padres, tutores o personas en cuyo poder se encuentren los niños, están obligados a proporcionarles como minimum de instrucción la materia de Moral y religión» (Weinberg, 1984). Hasta este momento, la materia de religión seguía presente en los contenidos fundamentales de la educación nacional. No obstante, en este mismo artículo se introdujo un principio de tolerancia religiosa. Se estableció la necesidad primordial de formar el carácter de los hombres por la enseñanza de la religión y las instituciones republicanas. En este sentido, el Consejo Nacional de Educación estaba obligado a respetar en la organización de la enseñanza religiosa las creencias de los padres de familia ajenos a la comunión católica.

Más tarde, en 1884, la Ley 1420 sentó las bases del sistema educativo argentino, atendió aspectos como la matrícula escolar, el personal docente, la inspección técnica y administrativa de las escuelas, la dirección y administración de las escuelas públicas, las bibliotecas populares y las escuelas y colegios particulares. A partir de su promulgación, el Estado continuó desarrollando acciones trascendentales para la expansión y sostenimiento del Sistema de Instrucción Pública, sobre todo en el nivel primario. Durante este periodo también se generaron debates a favor y 
en contra del carácter laico de la educación pública. Cabe mencionar que esta Ley no suprimió la enseñanza religiosa, pero sí estableció lineamientos que delimitarían su impartición en la escuela pública. El artículo $8^{\circ}$ estableció que no se podía impartir educación religiosa dentro de los horarios escolares por los ministros autorizados de los diferentes cultos a los niños de su respectiva comunión y antes o después de las horas de clase.

Después de su promulgación, se escucharon diversas críticas en contra del artículo 8 de la Ley 1420. Algunos puntos de vista cercanos a la tradición religiosa católica sostenían que esta ley era anticonstitucional, anticatólica, antipedagógica, antidemocrática, antihumana y, sobre todo, antiargentina por ir en contra de toda la tradición (Furlong, 1957). Los argumentos en contra de la Ley 1420 por parte de representantes de la Iglesia católica se guiaban bajo el supuesto que la educación pertenece a la familia por derecho natural y originario acordado por Dios, bajo esta óptica, el Estado no tenía derechos originarios sobre la educación y su acción más bien era la de suplir y completar la acción de la Iglesia y de las familias abriendo escuelas siempre dentro de las normas acordes al espíritu de la Iglesia católica. La Ley 1420 no suprimió la enseñanza religiosa, ni tampoco prohibió su impartición. De hecho, permitió que se continuara impartiendo en escuelas públicas, pero ya no como una materia de carácter obligatorio. La nueva disposición admitió que la educación religiosa pudiera ser confesional en virtud de que la imparten ministros de culto autorizados y no los profesores de las escuelas. Al mismo tiempo, la ley dejaba abierta la posibilidad de admitir la existencia de diferentes tipos de afiliaciones religiosas, pero en ese periodo había una clara hegemonía católica. Por último, también se establecía una regulación por parte del Estado para autorizar a los ministros de culto a impartir educación religiosa. La aplicación de la Ley 1420 tuvo problemas organizativos entre los gobiernos provinciales y el gobierno nacional (Aguerrondo y Rosas, 2006).

En el caso de Brasil, la educación religiosa ha sido un tema de debate desde el periodo colonial. Cuando el país permanecía como una colonia de Portugal, la hegemonía e influencia de la Iglesia se reflejó de forma clara en la Constitución ya que era la religión oficial y además de tenía subsidios y amparo del poder político. Todavía el decreto $\mathrm{n}^{\mathrm{o}} 7.247$ del 19 de abril de 1879 que reformó la enseñanza primaria y secundaria en el municipio de Côrte y la enseñanza superior en todo el imperio, mantuvo la instrucción moral y religiosa como parte de los contenidos 
educativos en las escuelas primarias de primer grado. Sin embargo, el artículo $4^{\circ}$ introdujo un principio de tolerancia para los alumnos no católicos (acatholicos) puesto que, por ley, no estaban obligados a asistir a las clases de instrucción religiosa. Adicionalmente, este tipo de instrucción debía impartirse en días determinados de la semana y siempre antes o después de las horas destinadas a otras disciplinas.

Fue así que el Estado comenzó a establecer lineamientos y controles específicos sobre la instrucción pública, regulando los tiempos y las formas para impartir la instrucción religiosa. Ahora, el artículo 25 de este mismo decreto, introdujo la posibilidad de que los académicos, directores, empleados y docentes de enseñanza primaria y secundaria pudieran prestar juramento conforme a su confesión religiosa o bien solo emitir la promesa de cumplir con sus deberes y funciones a todos los que por motivos religiosos estuvieran imposibilitados de prestar dicho juramento. Este cambio en cuanto a la forma y el protocolo que seguían los funcionarios públicos adquirió un valor cívico.

En síntesis, la educación laica estuvo estrechamente relacionada con el impacto de los procesos y debates universales que fueron especialmente significativos en tiempos y periodos de cambios trascendentales para las sociedades latinoamericanas. Al mismo tiempo que se pueden observar problemas y encrucijadas propiamente locales. En un marco general de conflictos internos y externos en cada uno de los países de América Latina, surgieron controversias sobre cómo se debía atender la cuestión de la enseñanza en cada país. Este tipo de cambios generaron muchas más críticas por parte de sectores académicos conservadores que, a su vez, eran simpatizantes de la educación religiosa y, por lo tanto, este tipo de cambios implicaban una ruptura en relación con los valores tradicionales que debía promover la escuela.

\section{Formas convergentes de la emergencia de la educación laica}

A partir de las independencias políticas de los Estados latinoamericanos y hasta finales del siglo XIX se desarrollaron cambios importantes en las relaciones Estado-Iglesia que modificaron también la forma en que se había conceptualizado la instrucción pública. En un primer momento se buscó propagar conocimientos prácticos acordes con la moral cristiana, la universalidad para formar ciudadanos católicos, creyentes de una misma fe, de una sola forma de pensamiento y no se toleraba 
ningún tipo de creencia distinta; en cierta medida, este tipo de instrucción era excluyente. Posteriormente, el control del Estado se fue ampliando de forma gradual sobre la instrucción primaria, lo que generó tenciones de diversos niveles en cada país.

El liberalismo político del siglo XIX se caracterizó por buscar la separación entre el Estado y la Iglesia. Las normas constitucionales en Argentina, Brasil y México se reajustaron para garantizar, en mayor o menor medida, el ejercicio de los derechos y libertades. Poco a poco se fueron omitiendo los contenidos religiosos apegados a la moral cristiana mediante disposiciones oficiales como fue el caso de México y, posteriormente también las escuelas oficiales en Argentina y Brasil ya no integraron la enseñanza religiosa dentro de sus contenidos curriculares.

Esto derivó en debates trascendentales sobre el control de la educación y, más tarde, se tradujo en la incorporación de la instrucción religiosa dentro del orden moral, pero ya no como un elemento sustantivo de la identidad nacional. Esta separación se reforzó con la promulgación de leyes reglamentarias entre 1861 y 1873 en los tres países. A través de estas medidas continuaron con la premisa de regular las actividades educativas de los agentes privados. Al mismo tiempo, se comenzó a implantar una regulación en la que la religión se fue desplazando del centro de toda la enseñanza impartida en las escuelas a cargo del Estado. De hecho, la influencia de la Iglesia católica también fue perdiendo peso a causa de la creación de un nuevo marco regulatorio en las instituciones educativas.

Los cambios en la legislación muestran que no es pertinente conceptualizar a la educación laica como una construcción por decreto. De hecho, estos cambios muestran la dificultad que presentaron Argentina, Brasil y México en cuanto a temas como la definición de los contenidos educativos en las leyes reglamentarias en la configuración del régimen de laicidad educativa. Durante la segunda mitad del siglo XIX, la religión ya no se integró como parte fundamental de las materias de enseñanza obligatoria. Estas omisiones muestran un primer intento por relegar a la religión de la escuela pública. Tanto en México (1861) como en Argentina (1873), un primer paso que se dio con relación al proceso de laicización de la enseñanza fue la omisión de los contenidos religiosos. En el caso de Brasil estas materias no desaparecieron, de hecho, seguían formando parte del currículum, pero se introdujo una variante que posibilitó que los alumnos ajenos a la comunión católica quedaran exentos de la obligación de frecuentar los salones de instrucción religiosa para 
obtener el grado de bachiller en letras. Esta medida fue muy similar a lo posteriormente se pondría en la Ley 1420 en Argentina, lo que implicaba que tampoco se consideraba como una materia obligatoria.

El alcance de las primeras disposiciones que comenzaron a configurar el régimen de laicidad educativa, en realidad fue muy limitado en términos geográficos. En términos generales estas medidas se circunscribían solo a determinas provincias. No obstante, es importante señalar que antes de la promulgación de las leyes generales y decretos no se tomaba en cuenta el derecho de los individuos, tampoco se consideraban las diferencias y divergencias de concepciones religiosas.

\section{Referencias bibliográficas}

Aguerrondo, I., y Rosas, M. (2006). Educación en la Argentina: qué pasó en los' 90. Buenos Aires, Argentina: Papers.

Arredondo, M. y Gonzales, R. (2015). De la secularización a la laicidad educativa en México. Historia de la Educación. Anuario, 15(2), 140167.

Barroso, J. (1867). A instruç̧ão pública no Brasil. Río de Janeiro, Brasil: BL Garnier.

Campobassi, J. (1961). Laicismo y catolicismo en la educación pública argentina. Buenos Aires, Argentina: Gure.

Cárdenas Ayala, E. (2015). El fin de una era: Pío IX y el Syllabus. Historia Mexicana, 65(2), 719-746.

Carvalho, J. (1995). Desenvolvimiento de la ciudadania en Brasil (Sección de obras de historia Serie Ensayos). México, México: Fondo de Cultura Económica.

Cunha, L. A. (2017). A educação brasileira na primeira onda laica: do Império à República. Río de Janeiro, Brasil: edición del autor.

Delgado Criado, B. (1992). Historia de la educación en España y América. Madrid, España: Morata.

Dublán, M. y Lozano, J. (1876). Legislación mexicana: o colección completa de las disposiciones legislativas expedidas desde la independencia de la República (Vol. 4).

Estrada, D. (1984). La educación ilustrada, 1786-1836: Educación primaria en la ciudad de México. México, México: Colegio de México. 
Furlong, G. (1957). La tradición religiosa en la escuela argentina: exposición histórica. Buenos Aires, Argentina: Theoria.

González Villarreal, R. y Arredondo, A. (2017). 1861: la emergencia de la educación laica en México. Historia Caribe, 12 (30), 25-49.

Martínez Paz, F. (1973). El sistema educativo nacional: Formación, desarrollo, crisis. San Miguel de Tucumán, Argentina : Fundación Banco Comercial del Norte.

Miranda, R. (1981). La descentralización del sistema escolar argentino: en el nivel de las relaciones nación-provincias. Rosario, Argentina: Instituto Rosario de Investigaciones en Ciencias de la Educación.

Newland, C. (1991). La educación elemental en Hispanoamérica: Desde la independencia hasta la centralización de los sistemas educativos nacionales. The Hispanic American Historical Review, 71(2), 335-364.

Sábato, H. (1999). Ciudadanía politica y formación de las naciones: Perspectivas históricas de América Latina (Serie Estudios. Sección de obras de historia). México, México: Fideicomiso Historia de las Américas El Colegio de México Fondo de Cultura Económica (FCE).

Staples, A. (1988). La lectura y los lectores en los primeros años de vida independiente. Historia de la lectura en México, 94-126.

Thornton, M.C. (1948). The Church and freemasory in Brazil, 1872-1875. A study in regalism. Washington, Estados Unidos: Faculty of the Graduate School of Arts and Sciences of the Catholic University of America.

Vázquez, J. (1993). El federalismo mexicano 1823-1847. En Carmagnani, M. Federalismos latinoamericanos: México, Brasil, Argentina. México, México: Fondo de Cultura Económica.

Weinberg, G. (1984). Debate parlamentario, ley 1420, 1883-1884. Vol. I. Buenos Aires, Argentina: Centro Editor de América Latina.

Zorraquín, R. (1996) Historia del derecho argentino, Tomo II (1810-1969). Buenos Aires, Argentina: Perrot. 